\title{
Tritrophic interactions between cereals, aphids and parasitoids: Discrimination of different plant-host complexes by Aphidius rhopalosiphi (Hymenoptera: Aphidiidae)
}

\author{
Stephan G. MICHA, Susanne KISTENMACHER, GunNar MÖLCK and Urs WYSS* \\ Institute of Phytopathology, University of Kiel, 24118 Kiel, Germany
}

Key words. Tritrophic interaction, herbivore, parasitoid, aphid, oat, wheat, Sitobion avenae, Aphidius rhopalosiphi, olfactometer, semiochemicals, synomone, host finding behaviour

\begin{abstract}
The tritrophic interactions between two different plant-host complexes, Avena sativa-Sitobion avenae, Triticum aestivum-S. avenae and the aphid parasitoid Aphidius rhopalosiphi were studied with respect to odour learning and recognition by the parasitoid. The orientation behaviour of females towards odours from either uninfested or aphid-infested oat or wheat plants was tested in a series of dual choice $Y$-tube olfactometer experiments. Female parasitoids had the opportunity to gain a single oviposition experience on either the oat-S. avenae or wheat-S. avenae complex before the experiment. In the first set of experiments, where $A$. rhopalosiph $i$ was reared on the oat-S. avenae complex, eight odour-bait combinations were tested. The females did not discriminate between uninfested oat and wheat. After oat complex experience, females responded to odours from the oat complex, but not to odours from the wheat complex. Consequently, in a direct comparison the oat complex was preferred over the wheat complex. After wheat complex experience, the parasitoid's orientation responses gave a different picture. Both, the wheat complex and the oat complex, were then shown to be equally attractive. Hence, in direct comparison no preference was recorded between the oat and wheat complexes. In a second set of experiments, where $A$. rhopalosiphi was reared on the wheat- $S$. avenae complex, a possible influence of any pre-adult or emergence-related host plant experience could be excluded as the same results were obtained as before. At first glance the responses towards the different odour baits seem inconsistent. However, the results may be explained using a simple model with two key odour components.
\end{abstract}

\section{INTRODUCTION}

Parasitoid searching behaviour and efficiency is one of the major issues in theoretical and applied ecological research. Host location behaviour has evolved under the pressure to use available host location cues efficiently. Chemical senses are highly developed and extensively exploited by parasitoids for host location (Quicke, 1997). Numerous volatile chemicals are produced by the hosts themselves and also, in most cases, by their food plants. Therefore, olfactory perception has to be considered as the major sense involved in host location.

Parasitoids find themselves confronted with a complex mixture of different plants and herbivores in natural environments. Only some of these plant-herbivore combinations may provide hosts for parasitoid reproduction. As has been shown in numerous studies, parasitoids employ very specific odour characteristics of the plants to distinguish between suitable and unsuitable plant-host combinations.

Herbivore attack very often induces the release of specific volatiles that guide predators or parasitoids in their search for prey or host, as first demonstrated by Dicke \& Sabelis (1988) and Turlings et al. (1990). These information providing chemicals, termed synomones in order to reflect their mutual beneficial effects (Nordlund, 1981), convey highly specific information about the plant-host complex (e.g. Vet et al., 1991; Vet \& Dicke, 1992; Geervliet et al., 1997). The important role of aphidinduced plant volatiles in mediating the foraging behaviour of aphid parasitoids has been well demonstrated in recent years by using Y-tube olfactometers and wind tunnel trials (e.g. Guerrieri et al., 1993, 1999; Micha, 1995; Du et al., 1996, 1998; Powell et al., 1998; Mölck et al., 1999).

The behavioural plasticity of parasitoids is also strongly related to the environmental variability in space and time. Learning ability, especially in the context of olfactory orientation, has been extensively demonstrated (reviewed in Vet et al., 1995). Parasitoids adapt to the changing availability of hosts on different plant species. After contacting hosts and/or plants, the behavioural response of the parasitoid towards the experienced stimuli is often considerably changed. Parasitoids will be attracted by that plant odour which was learned during oviposition into a suitable host (Vet \& Groenewold, 1990) due to either sensitization or associative learning (Turlings et al., 1993). Learning of synomones has recently been described for several plant-aphid-parasitoid systems (e.g. Grasswitz \& Paine, 1993; Grasswitz, 1998; Micha \& Wyss, 1995; Du et al., 1997; Poppy et al., 1997; Mölck et al., 1999), but the mechanisms involved are still not fully understood.

The aim of our study was to test the ability of aphid parasitoids to distinguish between plant-host complexes

\footnotetext{
* Correspondence Professor Dr. U. Wyss, Institut für Phytopathologie, Universität Kiel, Hermann-Rodewald-Str. 9, D-24118 Kiel, Germany.; e-mail: uwyss@phytomed.uni-kiel.de
} 
of closely related host plants infested with the same host species. In order to test the hypothesis that the parasitoids will predominately respond to the odours of plant-host complexes which they have learned as adults during an oviposition experience, a tritrophic model system, consisting of two different cereal plants with one aphid and one parasitoid species, was chosen. The results obtained show that more subtle processes during learning and/or recognition are involved that may produce unpredictable behavioural responses.

\section{MATERIAL AND METHODS}

\section{Rearing}

The tritrophic system consisted of the parasitoid Aphidius rhopalosiphi De Stefani-Perez, which was reared on the grain aphid (Sitobion avenae L.) on either oat (Avena sativa $\mathrm{L} . \mathrm{cv}$ Bojar) or wheat (Triticum aestivum L. cv. Troll). Parasitoids and aphids were reared on these plants at a $16 \mathrm{~L}: 8 \mathrm{D}$ photoperiod, $20^{\circ} \mathrm{C}\left( \pm 1^{\circ} \mathrm{C}\right)$ and at $60-70 \%$ r.h. in climatic chambers.

For parasitoid cultures, pots with one-week-old oat or wheat plants were infested with aphids of various stages and covered with an acrylic cylinder. On the same day, these rearing units were inoculated with parasitoid females. After 10 days, all aphid mummies were removed from the plants and transferred to a pot filled with plaster and covered with an acrylic cylinder. In these emergence units without any plant or aphid material, parasitoid females could feed ad libitum on sucrose solution (15\%) and mate freely with any males present.

\section{Standardization of plants and parasitoids}

Cereal plants were grown in groups of 10-20 in pots $(100 \mathrm{~mm}$ in diameter) with standard soil (Type ED 73, Patzke, Uetersen, Germany). Experimental plants were used about 2-3 weeks after sowing, having reached their 2- or 3-leaf stage. Plant infestation was initiated with 50 aphids of various stages per pot. Pots were then covered with an acrylic cylinder $(200 \mathrm{~mm}$ in height) and aphids could distribute themselves freely over the plants within a pot. After 6-8 days infestation levels varied on average between 40 and 60 aphids per plant. Experimental plant baits consisted of 10 leaf tips ( $60 \mathrm{~mm}$ in length) cut carefully with a razor blade just before the start of the experiments. Care was taken that the aphids remained undisturbed on these cut leaves.

One day before the experiment, $A$. rhopalosiphi females were removed from the emergence units and separated into groups of 10 individuals per acrylic vial $(100 \mathrm{~mm}$ in length, $18 \mathrm{~mm}$ internal diameter) provided with sucrose solution on a cotton wool plug. The age of these experimental parasitoid females was then 2-5 days. Before being used in the Y-tube olfactometer experiments, groups of parasitoids were treated differently on the same day with respect to plant-host experience. Prior to the experiments early in the morning, single females were introduced into acrylic vials containing leaf tips of either oat or wheat infested with $S$. avenae. Immediately after a single oviposition attack, the parasitoids were removed and placed back into another acrylic vial. One to two hours later, single females were tested in Y-tube olfactometer trials.

\section{Olfactometer bioassay}

An all-glass Y-tube olfactometer (after Read et al.,1970) was used in order to test the orientation responses of parasitoid females towards volatiles of the different plant-herbivore complexes. An air stream, generated by compressed air, was first dried with silica gel, then purified with charcoal filters and molecular sieves (A5) and finally passed through distilled water.
Bait glasses containing the odour sources were connected to the $Y$-arms. Bait chambers were separated by porous glass plates from those parts that were accessible to the parasitoids. After passing through the bait glasses, the adjusted air streams $\left(10.5 \pm 1.5 \mathrm{ml} \mathrm{min}^{-1}\right)$ entered into both $\mathrm{Y}$-arms and into the base arm where the two odour streams mixed. External light was excluded from the experimental chamber that was diffusely illuminated from above with four fluorescent tubes behind a white acrylic screen.

Females were released into the olfactometer by connecting a glass vial with a female to the exhaust end of the base arm. The position and movements of the female were observed for a maximum of $10 \mathrm{~min}$. Once it had reached the end of one Y-arm (i.e. the porous glass plate), the experiment was stopped, the female was removed and its choice recorded. After half of the number of the females per experience treatment had been tested, the Y-body was cleaned, the baits were renewed and their positions were exchanged to avoid any bias through side preferences of the parasitoids. After completing all replicates $(N=59$ to 60$)$ of one experiment, which was conducted over a series of days, all observed parasitoid decisions therein were pooled and statistically analysed against even distributions using the goodnessof-fit test. Distributions were regarded as indicating parasitoid attraction towards one bait if significantly different from an equal distribution with $\mathrm{p}<0.05(*),<0.01(* *),<0.001\left(^{* * *}\right)$.

\section{Experimental design}

The experiments had three different variables: plant-host complex for parasitoid rearing, oviposition experience of parasitoid, and plant bait odours offered in the Y-tube olfactometer. In the first set of experiments, all parasitoids were reared on the oat- $S$. avenae complex. In a second set of experiments, the parasitoids were reared on the wheat- $S$. avenae complex for several generations to test a possible influence of any pre-adult or emergence-related host plant experience.

In the first set of experiments (Fig. 1), all parasitoids had a single oviposition experience on either $S$. avenae-infested oat or infested wheat before the test. Four different bait combinations were tested as follows: uninfested oat vs. uninfested wheat, uninfested oat vs. infested oat, uninfested wheat vs. infested wheat and finally infested oat vs. infested wheat. In the second set of experiments (Table 1), only three selected bait combinations were tested in order to be compared with the results of the first set of experiments.

\section{RESULTS}

In the first set of experiments (all parasitoids reared on oat), parasitoids with aphid oviposition experience on oat (oat-experienced parasitoids) did not discriminate between the odours of uninfested oat and wheat (Fig. 1A). The same was true for wheat-experienced parasitoids (Fig. 1B). Oat- and wheat-experienced parasitoids were attracted to aphid-infested oat versus uninfested oat (Fig. 1C, D). Oat-experienced parasitoids did not discriminate between aphid-infested wheat versus uninfested wheat (Fig.1 E), whereas wheat-experienced parasitoids were attracted to aphid-infested wheat versus uninfested wheat (Fig. 1F). Oat-experienced parasitoids were attracted to aphid-infested oat versus aphid-infested wheat (Fig. 1G), whereas wheat-experienced parasitoids did not discriminate between aphid-infested wheat and aphid-infested oat (Fig. 1H).

In the second set of experiments (all parasitoids reared on wheat), only the three main experience-bait variants D, 
Orientation responses of $A$. rhopalosiphi females reared on an oat - $S$. avenae complex after a single oviposition experience on this complex
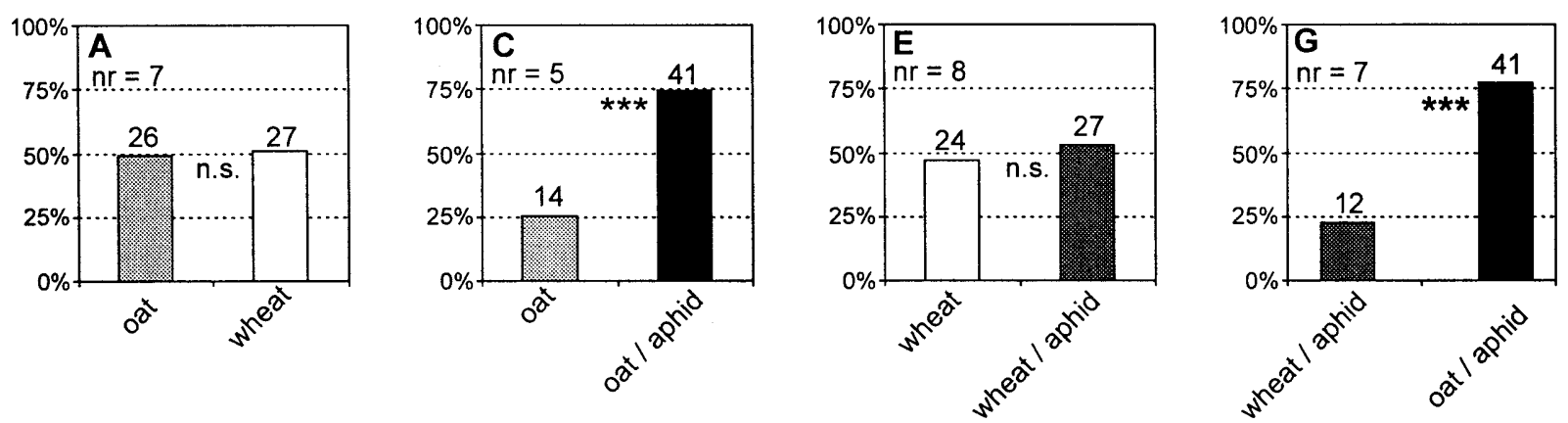

Orientation responses of $A$. rhopalosiphi females reared on an oat - $S$. avenae complex after a single oviposition experience on a wheat - $S$. avenae complex
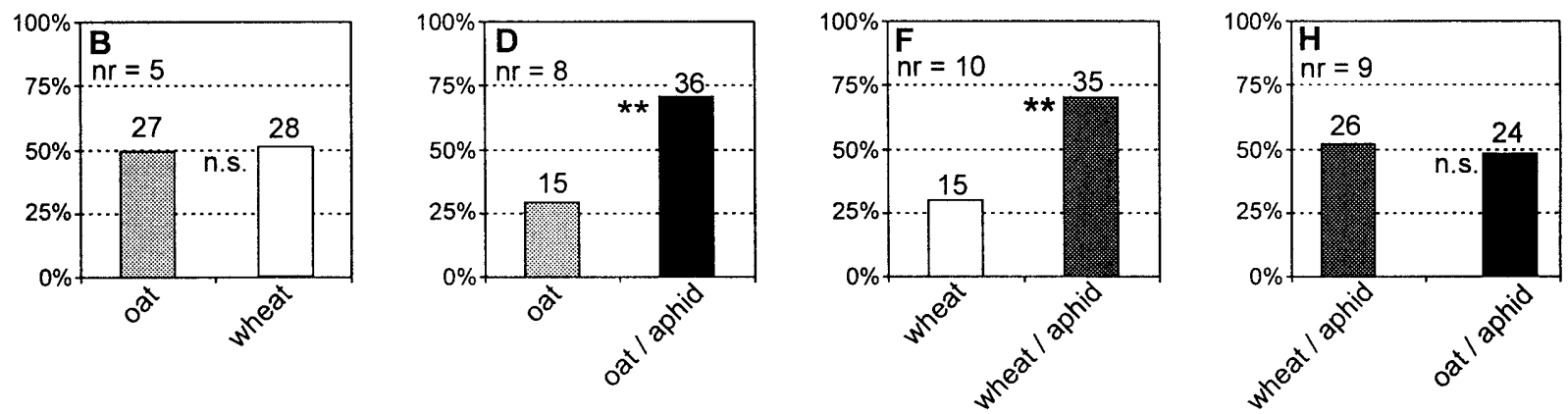

Fig. 1. Y-tube olfactometer results from A. rhopalosiphi females reared on the oat-S. avenae complex, after a single oviposition experience on this complex (upper graphs: A, C, E, G) or after a single oviposition experience on the wheat-S. avenae complex (lower graphs: B, D, F, H). Each graph represents the distribution of 59 to 60 parasitoid females for the two offered baits. $* *=$ $\mathrm{p}<0.01 ; * * *=\mathrm{p}<0.001 ; \mathrm{n} . \mathrm{s} .=$ not significantly different $(\mathrm{p} \geq 0.05) ; \mathrm{nr}=$ number of females that did not respond to the odour cues.

E and $\mathrm{H}$ were tested (Table 1). In all three cases the parasitoids showed the same responses as parasitoids reared on oat (Table 1).

TABLE 1. Responses of Aphidius rhopalosiphi females towards odours of different baits in dual choice Y-tube olfactometer experiments. The females were reared on a wheat- $S$. avenae complex and then tested after a single oviposition experience on this complex (variants $\mathrm{D}, \mathrm{H}$ ) or on an oat-S. avenae complex (variant $\mathrm{E}$ ). $\mathrm{N}=$ number of females with a response; $\mathrm{n}$ $=$ number of females without a response.

\begin{tabular}{lccccccc}
\hline $\begin{array}{l}\text { plant-host complex } \\
\text { experienced }\end{array}$ & \multicolumn{2}{c}{ oat - S. avenae } & \multicolumn{3}{c}{ wheat $-S$. avenae } \\
\hline baits: & variants & $\mathrm{N}$ & $\mathrm{n}$ & variants & $\mathrm{N}$ & $\mathrm{n}$ \\
\hline $\begin{array}{l}\text { uninfested oat } \\
\begin{array}{l}\text { oat infested with } \\
\text { S. avenae }\end{array}\end{array}$ & $\begin{array}{c}\text { not } \\
\text { tested }\end{array}$ & & D & 18 & 5 \\
\hline
\end{tabular}

\begin{tabular}{|c|c|c|c|c|c|c|}
\hline $\begin{array}{l}\text { uninfested wheat } \\
\text { wheat infested with } \\
\text { S. avenae }\end{array}$ & $\mathbf{E}$ & $\begin{array}{c}25 \\
27 \text { n.s. }\end{array}$ & 7 & $\begin{array}{l}\text { not } \\
\text { tested }\end{array}$ & & \\
\hline $\begin{array}{l}\text { wheat infested with } \\
\text { S. avenae }\end{array}$ & not & & & $\mathbf{H}$ & & 7 \\
\hline $\begin{array}{l}\text { oat infested with } \\
\text { S. avenae }\end{array}$ & tested & & & & $27 \mathrm{n} . \mathrm{s}$ & \\
\hline
\end{tabular}

$*=p<0.05 ;$ n.s. $=$ not significantly different $(p \geq 0.05)$

\section{DISCUSSION}

As cut leaves were used in our experiments, they may have emitted some additional damage-related volatiles. However, this side effect was accepted because preliminary results did not indicate that mechanical damage of that kind caused any significant changes in parasitoid response (Micha, 1995; Micha \& Wyss, 1995). This was confirmed by recent olfactometer experiments where parasitoid responses to intact potted plants and cut leaves were compared (unpubl.). The aphids themselves were also not attractive (Micha, 1995; Micha \& Wyss, 1995). Care was taken to avoid aphid disturbance, which can cause the release of alarm pheromone in cornicle secretions, which is attractive for some aphid parasitoids (Micha \& Wyss, 1996).

The results show that parasitoid orientation behaviour in the olfactometer is influenced by the odours emitted from the plant baits. The observed choice pattern gives a complex picture of parasitoid preferences for certain plant odours. The basic assumption is that parasitoids learn the odours which they perceive in association with a rewarding experience and that they will be attracted to these odours later. Consequently, uninfested oat and wheat plants should not be attractive for the parasitoids, uninfested alternatives should always be ignored and infested plants should be attractive to those parasitoids that have previously gained experience on the specific 
Odour components experienced on the oat - S.avenae complex: $A+x$
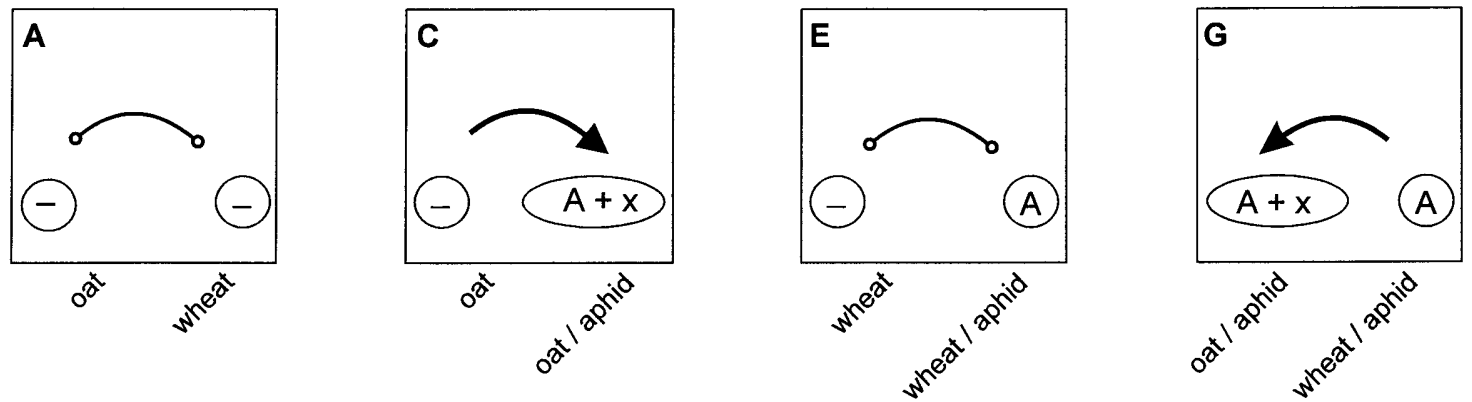

Odour components experienced on the wheat - S.avenae complex:A

(ignoring ' $x$ ')
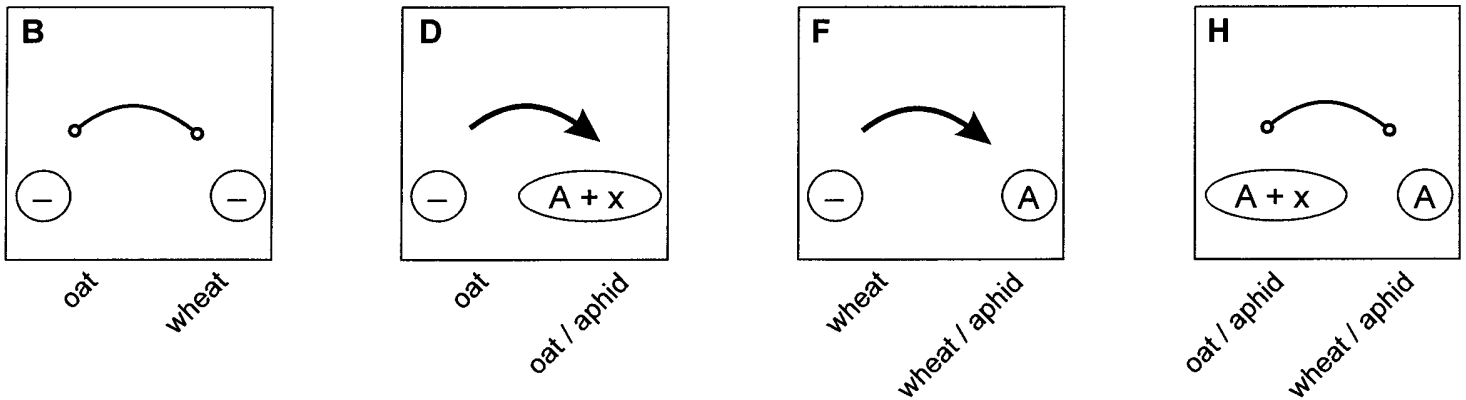

Fig. 2. Model for odour compositions in oat and wheat. Arrows represent significant orientations towards odour sources in the Y-tube olfactometer experiments (see Fig. 1). Letters "A" and " $x$ " are assigned to the two plant-host complexes in order to indicate the presence of hypothetical odour components that can explain the observed behavioural reactions of the parasitoids. See also text for further details.

plant-host complex (Fig. 1C, F). According to this hypothesis, the behavioural pattern of the parasitoids with wheat- $S$. avenae experience can only be understood under the assumption that infested oat and infested wheat have an odour in common that is attractive to wheatexperienced parasitoids. This shared odour characteristic should have been previously learned by the parasitoids.

A different picture appears when the results from the oat-S. avenae-experienced females are considered. These parasitoids did not respond to the wheat- $S$. avenae complex. It seems therefore that these females have not gained sufficient information from previous oviposition experience to allow them to recognize the wheat- $S$. avenae complex.

In order to explain this observed behavioural pattern, we propose a model to characterize the odour composition of the plants, using a minimum number of odour components (Fig. 2). No odours (-) are assigned to both uninfested plant species. A shared odour component " $\mathrm{A}$ " is assigned to both infested plant species. An additional component " $\mathrm{x}$ " is assumed in the oat-S. avenae complex. The recognition of infested oat and infested wheat by wheat-experienced wasps is based on component " $\mathrm{A}$ ". After learning " $A$ " as an exclusive indicator for a suitable plant-host complex, both, infested wheat and infested oat are attractive. No preference is shown between these two (Fig. $2 \mathrm{H}$ ) as the additional component " $\mathrm{x}$ ", occurring in the odour blend of infested oat, is meaningless to these females and is consequently ignored. If the females learned both components ("A $+\mathrm{x}$ ") in one plant-host complex (here in infested oat) then only " $\mathrm{A}+\mathrm{x}$ " is attractive (Fig. 2C, G) but not "A" alone (Fig. 2E).

In additional tests, we examined if pre-adult or emergence-related host plant experiences can influence parasitoid decisions - as was reported by Wickremasinghe \& van Emden (1992) and van Emden et al. (1996). Three experiments were therefore repeated with parasitoids that had been reared on the wheat- $S$. avenae complex for several generations. The results of these experiments did not differ from the results of oat-reared females, which indicates that in this case pre-adult experience is much less important for host-plant complex recognition than oviposition experience. The possibility that the indifference towards wheat baits was caused by rearing the parasitoids on the oat-S. avenae complex can therefore be excluded. This is not necessarily at variance with the chemical legacy hypothesis supported by the findings of van Emden et al. (1996). Contact to suitable aphid hosts may provide more recent and thus more important information to the foraging female than the chemical information conveyed through the mummy case from which it had emerged. The stimulus of a successful oviposition to learn the present odour blend may therefore override the effects of conditioning (Poppy et al., 1997) during emergence.

Before conducting these experiments, we assumed that parasitoids would choose only those plant-host complexes on which they had previously gained oviposition experi- 
ence. Generally, we expected that parasitoids which previously had successful host encounters on a particular plant, would memorize the chemical information about this plant, i. e. its odour composition (Vet \& Dicke, 1992). Later they would employ this information to recognize the plant as a potential source of hosts. However, the results presented here do not fully support this model.

We therefore developed a simple mechanistic model based on our experimental observations. Of course it cannot be concluded that oat and wheat odour recognition by the parasitoids is based on only two components. Wheat and oat emit much more complex odour blends and presumably parasitoids perceive more than only two of those components. In order to finally solve the mechanisms of learning and recognition that determine the observed phenomenon of plant-host complex preferences by $A$. rhopalosiphi, the chemicals involved must be identified. Our model may provide some additional information helping in the search for key components of plant odours which influence parasitoid host location behaviour.

ACKNOWLEDGEMENTS. The authors gratefully acknowledge the financial support of the Deutsche Forschungsgemeinschaft (DFG, Wy 9/14-3). Experimental work was conducted with the help of M. Wittke.

\section{REFERENCES}

Dicke M. \& SABelis M.W. 1988: How plants obtain predatory mites as bodyguards. Neth. J. Zool. 38: 148-165.

Du Y.-J., Popry G.M. \& Powell W. 1996: Relative importance of semiochemicals from first and second trophic levels in host foraging behavior of Aphidius ervi. J. Chem. Ecol. 22: 1591-1605.

Du Y.-J., Poppy G.M., Powell W. \& Wadhams L.J. 1997: Chemically mediated associative learning in the host foraging behavior of the aphid parasitoid Aphidius ervi (Hymenoptera: Braconidae). J. Insect Behav. 10: 509-522.

Du Y.-J., Poppy G.M., Powell W., Pickett J.A., Wadhams L.J \& WoODCOCK C.M. 1998: Identification of semiochemicals released during aphid feeding that attract parasitoid Aphidius ervi. J. Chem. Ecol. 24: 1355-1368.

Geervliet J.B.F., Posthumus M.A., Vet L.E.M. \& Dicke M 1997: Comparative analysis of headspace volatiles from different caterpillar-infested or uninfested food plants of Pieris species. J. Chem. Ecol. 23: 2935-2954.

Grasswitz T.R. \& Paine T.D. 1993: Effect of experience on inflight orientation to host-associated cues in the generalist parasitoid Lysiphlebus testaceipes. Entomol. Exp. Appl. 68 : 219-229.

Grasswitz T.R. 1998: Effect of adult experience on the hostlocation behavior of the aphid parasitoid Aphidius colemani Viereck (Hymenoptera: Aphidiidae). Biol. Control 12: 177-181.

Guerrieri E., Pennacchio F. \& Tremblay E. 1993: Flight behaviour of the aphid parasitoid Aphidius ervi (Hymenoptera: Braconidae) in response to plant and host volatiles. Eur. J. Entomol. 90: 415-421.

Guerrieri E., Poppy G.M., Powell W., Tremblay E. \& PennacCHIO F. 1999: Induction and systemic release of herbivoreinduced plant volatiles mediating in-flight orientation of Aphidius ervi. J. Chem. Ecol. 25: 1247-1261.
Micha S.G. \& Wyss U. 1995: Bedeutung von Pflanzenduftstoffen bei der Wirtssuche von Aphidius uzbekistanicus (Hymenoptera, Aphidiidae), eines Parasitoiden der Großen Getreideblattlaus (Sitobion avenae). Gesunde Pflanzen 47: 300-307.

Micha S.G. 1995: Die Bedeutung von Semiochemikalien im tritrophischen System aus Hafer (Avena sativa), Große Getreideblattlaus (Sitobion avenae) und Blattlausparasitoid (Aphidius uzbekistanicus). $\mathrm{PhD}$ thesis, University of Kiel, Germany, $150 \mathrm{pp}$.

MrchA S.G. \& WYss U. 1996: Aphid alarm pheromone (E)-Bfarnesene: a host finding kairomone for the aphid primary parasitoid Aphidius uzbekistanicus (Hymenoptera: Aphidiinae). Chemoecology 7: 132-139.

MöLCK G., Micha, S.G. \& WYSS U. 1999: Attraction to odour of infested plants and learning behaviour in the aphid parasitoid Aphelinus abdominalis. J. Plant Diseases and Protection 106: 557-567.

NoRDLUND D.A. 1981: Semiochemicals: A review of the terminology. In Nordlund D.A., Jones R.L. \& Lewis W.J. (eds): Semiochemicals, their Role in Pest Control. Wiley \& Sons, New York, pp. 13-28.

Poppy G.M., Powell W. \& Pennacchio F. 1997: Aphid parasitoid responses to semiochemicals - genetic, conditioned or learnt? Entomophaga 42: 193-199.

Powell W., Pennacchio F., Poppy G.M. \& Tremblay E. 1998: Strategies involved in the location of hosts by the parasitoid Aphidius ervi Haliday (Hymenoptera: Braconidae: Aphidiinae). Biol. Control 11: 104-112.

Quicke D.L.J. 1997: Parasitic Wasps. Chapman \& Hall, London, $470 \mathrm{pp}$.

Read D.P., Feeny P.P. \& Root R.B. 1970: Habitat selection by the aphid parasite Diaeretiella rapae (Hymenoptera: Braconidae) and hyperparasite Charips brassicae (Hymenoptera: Cynipidae). Can. Entomol. 102: 1567-1578.

Turlings T.C.J., Tumlinson J.H. \& Lewis W.J. 1990: Exploitation of herbivore-induced plant odors by host-seeking parasitic wasps. Science 250: 1251-1253.

Turlings T.C.J., Wäckers F.L., Vet L.E.M., LeWIS W.J. \& TuMLINSON J.H. 1993: Learning of host-finding cues by Hymenopterous parasitoids. In Papaj D.R. \& Lewis A.C. (eds): Insect Learning: Ecological and Evolutionary Perspectives. Chapman \& Hall, New York, pp. 51-78.

van Emden H.F., Sponagl B., Wagner E., Baker T., Ganguly S. \& Douloumpaka S. 1996: Hopkins' "host selection principle", another nail in its coffin. Physiol. Entomol. 21: 325-328.

Vet L.E.M. \& Groenewold A.W. 1990: Semiochemicals and learning in parasitoids. J. Chemical Ecol. 16: 3119-3135.

Vet L.E.M., WÄCKERS F.L. \& DiCKE M. 1991: How to hunt for hiding hosts: The reliability-detectability problem in foraging parasitoids. Neth. J. Zool. 41: 202-213.

VET L.E.M. \& Dicke M. 1992: Ecology of infochemical use by natural enemies in a tritrophic context. Annu. Rev. Entomol. 37: 141-172.

Vet L.E.M., Lewis W.J. \& CARdÉ R.T. 1995: Parasitoid foraging and learning. In Cardé R.T. \& Bell W.J. (eds): Chemical Ecology of Insects 2. Chapman \& Hall, New York, pp. 65-101.

Wickremasinghe M.G.V. \& VAN EMDEN H.F. 1992: Reactions of adult female parasitoids, particularly Aphidius rhopalosiphi, to volatile chemical cues from the host plant of their aphid prey. Physiol. Entomol. 17: 297-304.

Received January 6, 2000; accepted May 18, 2000 